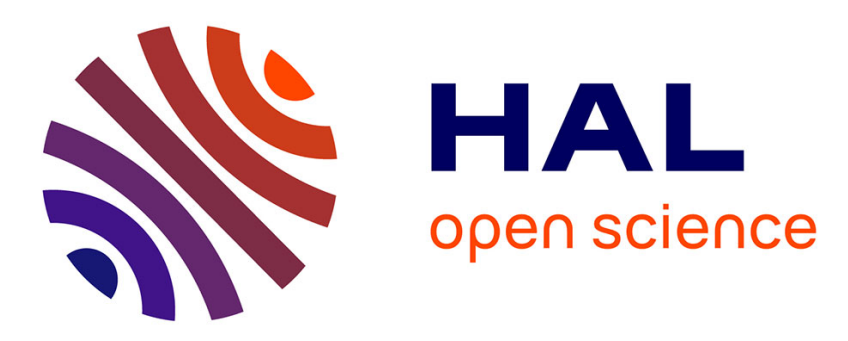

\title{
Utilisation d'un interféromètre de Michelson pour l'étalonnage des spectres obtenus à l'aide du spectromètre Fabry-Perot photo-électrique
}

\author{
H.T. Duong, S. Gerstenkorn, J.M. Helbert
}

\section{To cite this version:}

H.T. Duong, S. Gerstenkorn, J.M. Helbert. Utilisation d'un interféromètre de Michelson pour l'étalonnage des spectres obtenus à l'aide du spectromètre Fabry-Perot photo-électrique. Revue de Physique Appliquée, 1967, 2 (4), pp.249-252. 10.1051/rphysap:0196700204024900 . jpa-00242800

HAL Id: jpa-00242800

https://hal.science/jpa-00242800

Submitted on 1 Jan 1967

HAL is a multi-disciplinary open access archive for the deposit and dissemination of scientific research documents, whether they are published or not. The documents may come from teaching and research institutions in France or abroad, or from public or private research centers.
L'archive ouverte pluridisciplinaire HAL, est destinée au dépôt et à la diffusion de documents scientifiques de niveau recherche, publiés ou non, émanant des établissements d'enseignement et de recherche français ou étrangers, des laboratoires publics ou privés. 


\title{
UTILISATION D'UN INTERFÉROMÈTRE DE MIGHELSON POUR L'ÉTALONNAGE DES SPEGTRES OBTENUS A L'AIDE DU SPEGTROMÈTRE FABRY-PEROT PHOTO-ÉLEGTRIQUE
}

\author{
Par H. T. DUONG, S. GERSTENKORN, J. M. HELBERT, \\ Laboratoire Aimé-Cotton, Bellevue et G.E.A. (Saclay).
}

\begin{abstract}
Résumé. - L'étalonnage des spectres obtenus à l'aide du spectromètre photo-électrique Fabry-Perot ("Hypeac ") est réalisé à l'aide d'un interféromètre de Michelson du type Saunders. Par comparaison avec des mesures effectuées avec des techniques de radio-fréquence, sur la raie $\lambda=4077 \AA$, on montre qu'une précision de $10^{-4}$ peut être atteinte facilement à l'aide de ce dispositif.
\end{abstract}

Abstract. - To calibrate the spectrum given by the "Hypeac" (Fabry-Perot photoelectric spectrometer), we have used an auxiliary Michelson interferometer of the Saunders type. By comparison with measurements made by $\mathrm{R}$. H. Kohler using radio-frequencies techniques on the mercury line $\lambda=4077 \AA$, we show that a precision of $10^{-4}$ is easily attained.

1. Introduction. - Les méthodes utilisées jusqu'ici pour mesurer les distances entre composantes hyperfines enregistrées à l'aide du spectromètre FabryPerot photo-électrique peuvent être classées en trois groupes distincts :

a) Méthodes qui tendent à faire varier aussi linéairement que possible la pression (donc l'indice), à l'intérieur de l'enceinte du Fabry-Perot, en fonction du temps [1], [2], [3].

b) Utilisation d'un enregistreur $x, y$ [4], [5] : l'axe des y traduit l'intensité des raies spectrales, tandis que le mouvement dans la direction des $\mathrm{x}$ est assuré par l'intermédiaire d'un manomètre de conception spéciale dont la réponse est linéaire en fonction de la pression régnant dans l'enceinte contenant le FabryPerot.

c) Enregistrement simultané, en fonction du temps, du spectre donné par le Fabry-Perot et de la pression. L'enregistrement de la pression est obtenu par un moyen optique, en l'occurrence un second FabryPerot [6], [7] (Fabry-Perot de référence, fig. 1).

Les méthodes du premier groupe, les plus employées, parviennent à assurer, dans les meilleures conditions, une reproductibilité de $3 / 1000$ entre les différents ordres.

En ce qui concerne les méthodes du deuxième groupe, séduisantes a priori car elles éliminent le facteur temps, outre qu'elles nécessitent l'utilisation d'un enregistreur $x, y$, elles dépendent d'un organe mécanique; la reproductibilité est finalement fonctioin de la linéarité de la réponse du manomètre. Jusqu'ici,

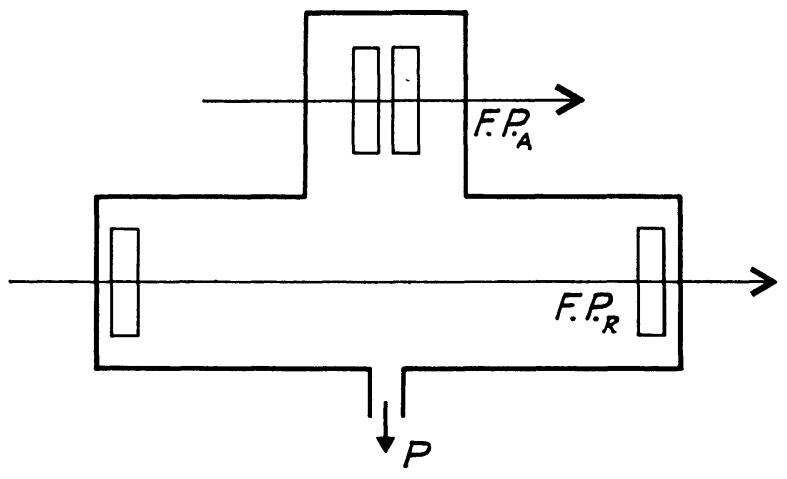

FIG. 1.

nous n'avons pas encore trouvé dans la littérature une publication donnant les performances ultimes de ce type d'appareil.

Enfin, les méthodes du troisième groupe nous semblent les plus prometteuses : en effet, la loi d'entrée d'air dans l'enceinte du Fabry-Perot peut être quelconque, pourvu toutefois que le pointé des pics à la précision voulue reste toujours possible.

Pour que l'étalonnage soit suffisamment serré, il faut que l'ordre $p$ d'interférence du Fabry-Perot de référence $\left(F . P_{R}\right.$, fig. 1) soit très élevé. Si l'on veut obtenir un pic du F. $P_{R}$ tous les $10 \mathrm{mK}$, il faut que la distance entre les lames soit égale à $50 \mathrm{~cm}$ dans le cas d'un Fabry-Perot plan et $25 \mathrm{~cm}$ dans le cas du Fabry-Perot sphérique [8]. Dans ce cas, la seule source donnant des raies suffisamment fines est un laser. Or, P. Connes nous a suggéré d'utiliser un interféromètre 
de Michelson, du type Saunders ( fig. $2 a$ ) à la place d'un Fabry-Perot, ce qui permet d'obtenir un étalonnage aussi serré que l'on veut, et ceci sans avoir besoin de laser.

2. Principe de l'étalonnage du spectre à l'aide de l'interféromètre de Michelson. - Dans l'enceinte où se trouve le Fabry-Perot, est placé un interféromètre de Michelson : l'un des bras du Michelson communique directement avec l'enceinte, l'autre bras est soit sous vide, soit maintenu à une pression constante proche de la pression atmosphérique. Le montage de cet interféromètre est celui préconisé par Saunders [9], la séparatrice et la compensatrice étant réali-

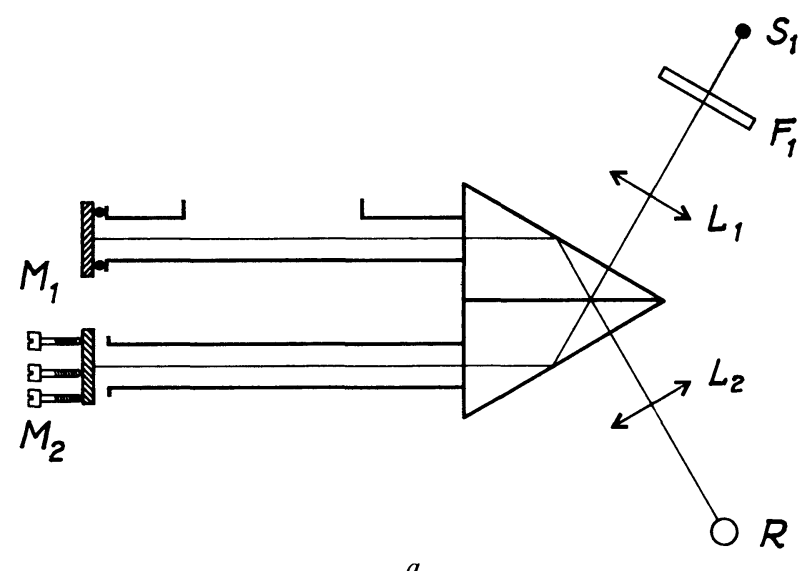

sées à l'aide d'un prisme de Kösters. Les deux miroirs $\mathrm{M}_{1}$ et $\mathrm{M}_{2}$ ( fig. $2 a$ ) sont réglés de telle sorte que l'on soit à la différence de marche zéro. Il est donc possible d'observer les interférences avec une source émettant des raies relativement larges : la plus simple est une lampe à mercure basse pression dont on isole la raie verte à l'aide d'un filtre.

Lorsqu'on fait varier l'indice de l'air $n$ dans l'enceinte où se trouve le Fabry-Perot, on observe dans l'interféromètre dont l'un des bras communique avec l'enceinte, soit des anneaux, soit des franges selon que les miroirs $M_{1}$ et $M_{2}$ sont parallèles ou font entre eux un petit angle. Dans tous les cas, la variation d'indice $\mathrm{d} n^{\prime}$ à l'intérieur de l'enceinte, pour passer d'un maximum à l'autre (anneau ou frange) dans le Michelson, est donnée par la relation $\mathrm{d} n^{\prime}=\lambda_{\mathrm{I}} / 2 L$ ( $\lambda_{\mathrm{I}}$ longueur d'onde passant à travers l'interféromètre, $L$ : longueur des bras du Michelson). Or, pour passer d'un pic à l'autre dans le Fabry-Perot, il faut effectuer une variation d'indice dn correspondant à un ordre du Fabry-Perrot, c'est-à-dire $\mathrm{d} n=\lambda_{\mathrm{FP}} / 2 e$ ( $\lambda_{\text {FP }}$ longueur d'onde analysée par le Fabry-Perot, $e$ distance entre les lames du F.P.).

Il en résulte que le nombre $m$ de franges que l'on peut observer grâce au Michelson pendant la variation d'indice $\mathrm{d} n$ correspondant à un ordre du Fabry-Perot est égale à :

$$
m=\mathrm{d} n / \mathrm{d} n^{\prime}=\frac{L}{e} \cdot \frac{\lambda_{\mathrm{FP}}}{\lambda_{\mathrm{I}}} .
$$

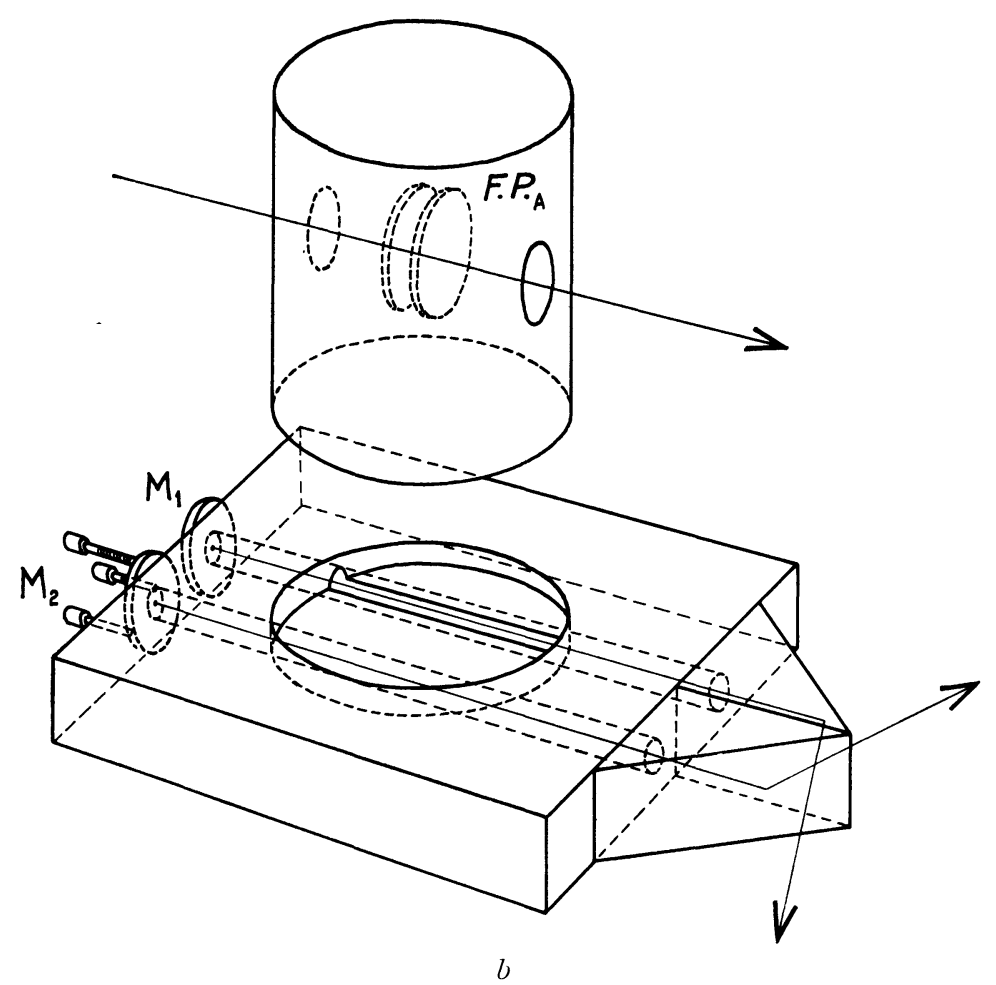

FIG. 2. 
Si l'on prend $L=300 \mathrm{~mm}, e=5 \mathrm{~mm}$, on obtient une soixantaine de franges environ (selon la valeur du rapport $\lambda_{\mathrm{FP}}\left(\lambda_{\mathrm{I}}\right)$.

Après avoir défini au mieux l'axe d'un pic donné par le Fabry-Perot, il suffit de repérer la position de cet axe par rapport à une frange donnée par le Michelson. Pour mesurer la distance séparant deux composantes, on compte le nombre de franges compris entre ces deux composantes auquel on ajoute les excédents fractionnaires.

3. Performances. - Pour tester notre montage, nous avons mesuré la structure hyperfine de la raie $4077 \AA$ $\left(6 s 6 p^{3} P_{1}-6 s 7 s^{1} S_{0}\right)$ du mercure à l'aide d'une source contenant 80,44 \% de l'isotope 201 (tableau I, fig. 3).

Grâce au travail de R. H. Kohler [10], la structure hyperfine du niveau ${ }^{3} P_{1}$ est connue avec une grande précision (tableau II, colonne 2). Dans la troisième colonne de ce tableau, nous avons converti les valeurs de R. H. Kohler (mesurées en $\mathrm{Mc} / \mathrm{s}$ ) en $\mathrm{mK}$ $\left(1 \mathrm{mK}=10^{-3} \mathrm{~cm}^{-1}\right)$. Pour ce faire, nous avons admis que la vitesse de la lumière $c$ est égale à $299793 \pm 1 \mathrm{~km} / \mathrm{s}$; il en résulte une incertitude relative de $3 \times 10^{-6}$ sur la valeur des structures hyperfines exprimées en $\mathrm{mK}$. Dans la quatrième colonne figurent les valeurs mesurées par W. G. Schweitzer [11] (méthode utilisée : jet atomique par absorption). Enfin, la cinquième colonne donne les résultats que nous avons obtenus.

Ce tableau montre que, si l'on considère l'erreur arithmétique moyenne, la précision de nos mesures est du même ordre de grandeur que celle donnée par la méthode utilisée par W. G. Schweitzer. L'erreur probable de nos mesures correspond à une précision de $10^{-4}$. L'accord entre nos résultats et ceux de R. H. Kohler pour la structure totale $(201 \mathrm{a}-201 \mathrm{c})$ est excellent et est de l'ordre de quelque $10^{-5}$ (l'épaisseur de notre Fabry-Perot a été mesurée à l'aide de la

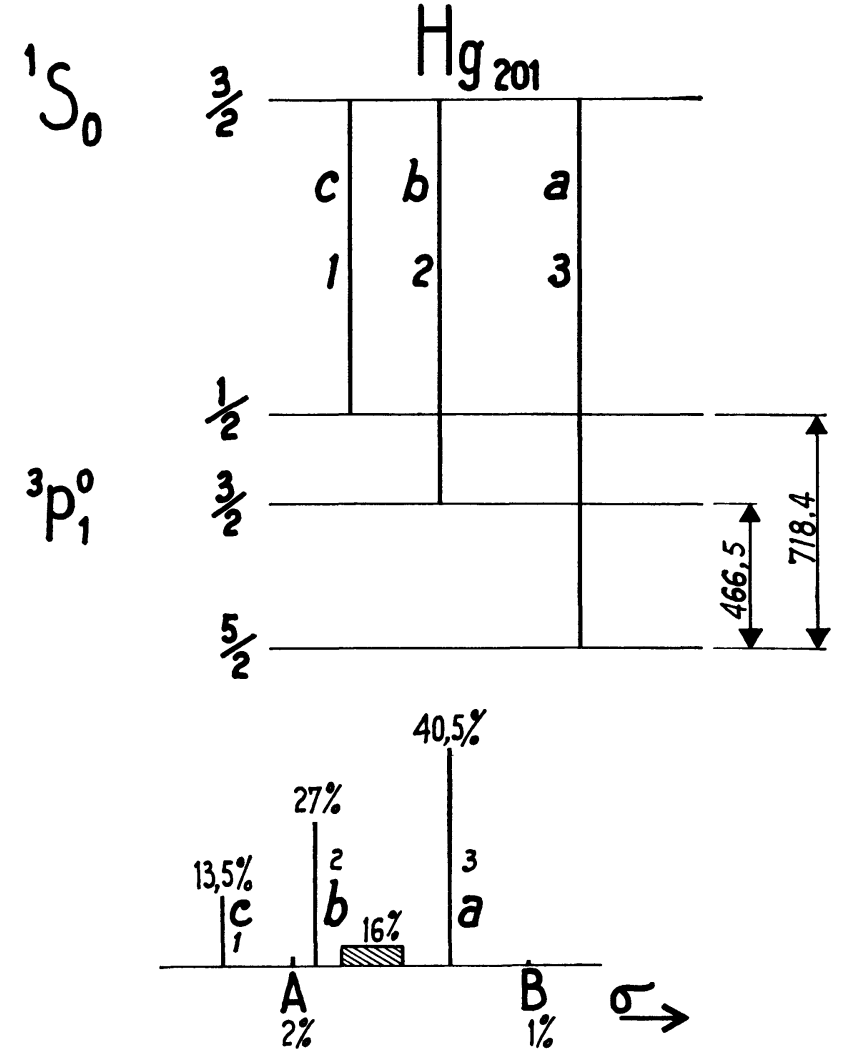

FIG. 3. $-\lambda=4077 \AA\left(6 s 6 p^{3} P_{1}-6 s 7 s^{1} S_{0}\right)$.

méthode des excédents fractionnaires avec une précision de $\left.2 \times 10^{-5} ; e=4,59975 \pm 0,0001 \mathrm{~mm}\right)$. L'accord est moins bon $\left(2 \times 10^{-4}\right)$ pour l'intervalle 201 a$201 \mathrm{~b}$. Ce moins bon accord est aisément explicable, car la composante $201 \mathrm{~b}$ est légèrement perturbée par la présence de la composante hyperfine A ( fig. 3) due à l'isotope 199.

\begin{tabular}{cccccccc}
\multicolumn{7}{c}{ TABLEAU I } \\
Isotope & 196 & 198 & 199 & 200 & 201 & 202 & 204 \\
$\frac{-}{\%}$ & - & - & - & - & - & - & - \\
& 0,05 & 1,63 & 2,95 & 5,89 & 80,44 & 7,95 & 1,14
\end{tabular}

TABLEAU II

\begin{tabular}{|c|c|c|}
\hline INTERVAL,LE & \multicolumn{2}{|c|}{ MESURES DE R. H. KOHLER [10] } \\
\hline $\begin{array}{l}\text { HYPERFIN } \\
\text { DU NIVEAU }{ }^{3} P_{1}\end{array}$ & $\mathrm{EN} \mathrm{Mc} / \mathrm{s}$ & $\mathrm{EN} \mathrm{mK}$ \\
\hline $\begin{array}{l}201 \mathrm{~b}-201 \mathrm{c} \\
201 \mathrm{a}-201 \mathrm{~b}\end{array}$ & $\begin{array}{r}7551,613 \pm 0,013 \\
13986,557 \pm 0,008\end{array}$ & $466,541 \pm 0,001$ \\
\hline $201 \mathrm{a}-201 \mathrm{c}$ & $21538,170 \pm 0,021$ & $718,435 \pm 0,002$ \\
\hline
\end{tabular}

MESURES DE

W. G. SCHWETTZER [11]

JET ATOMIQUE EN ABSORPTION

$466,40 \pm 0,34\left(^{1}\right)$

$718,17 \pm 0,51\left({ }^{1}\right)$
MESURES

PAR FABRY-PEROT E'T ETALONNAGE PAR MICHELSON (EMISSION)

$466,64 \pm 0,4\left({ }^{1}\right)$ $466,64 \pm 0,1(2)$ $718,42 \pm 0,4\left({ }^{1}\right)$ $718,42 \pm 0,1\left({ }^{2}\right)$

(1) Erreur arithmétique moyenne.

(2) Erreur probable. 


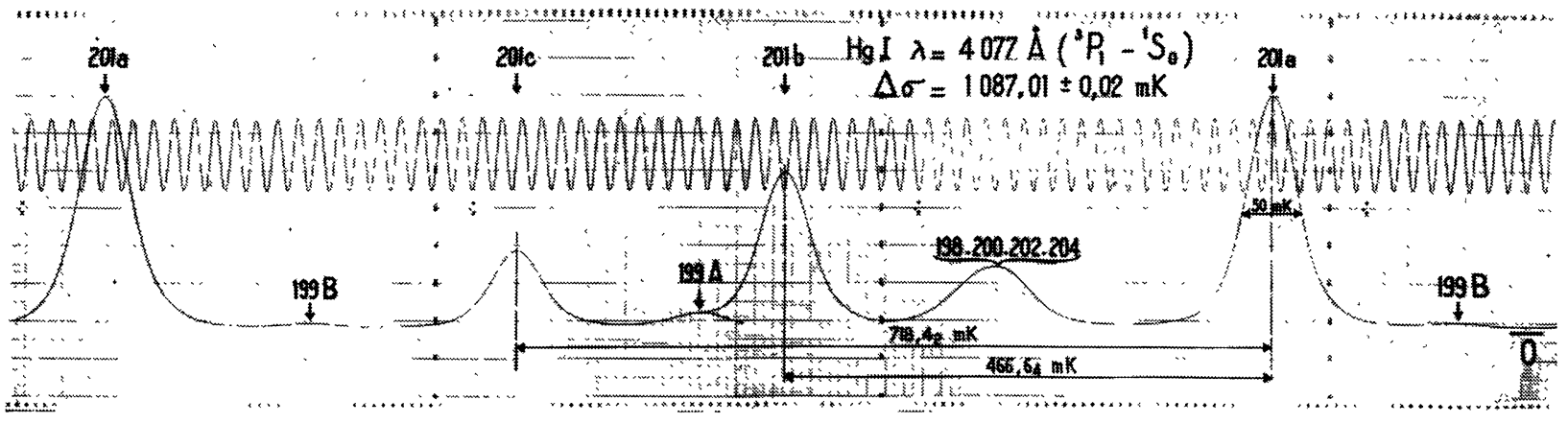

FIG. 4.

4. Conditions expérimentales. - La source que nous avons utilisée était un tube sans électrodes excité par haute fréquence, il contenait $1 \mathrm{mg}$ environ de mercure. Les mesures ont été effectuées à l'aide d'un montage du type " Hypeac " [12]; l'étalon FabryPerot était à « cales fixes » [13]. L'intervalle spectral libre $\Delta \sigma$ était égal à $1087,01 \pm 0,02 \mathrm{mK}$; la largeur des raies enregistrées est de l'ordre de $50 \mathrm{mK}$ et correspond donc à une finesse effective de 21 environ. Le rapport signal/bruit de nos enregistrements (fig. 4) nous permet de pointer les axes des composantes hyperfines avec une erreur maximum de $0,1 \mathrm{mK}$, c'est-àdire au 1/500 de la largeur de raie. A la précision de nos pointés, les composantes $201 \mathrm{a}$ et $201 \mathrm{c}$ sont symétriques depuis leur sommet jusqu'au sixième de leur hauteur. L'échelle réelle de nos enregistrements de la figure 4 est de $3,5 \mathrm{~mm}$ pour $1 \mathrm{mK} ; 0,1 \mathrm{mK}$ correspond donc à $0,35 \mathrm{~mm}$, ce que l'on peut aisément apprécier. La stabilité de la source est telle que les intensités des composantes hyperfines $201 \mathrm{a}, 201 \mathrm{~b}$ et $201 \mathrm{c}$ sont respectivement proportionnelles aux nombres $3,2,1$ (à $0,5 \%$ près) conformément à la théorie. Nous avons donc là une indication que les erreurs entraînées soit par des fluctuations de la source, soit par la présence d'auto-absorption ne sont pas à craindre.

5. Avantage du montage. - 1) Stabilité. - La construction de l'interféromètre est telle qu'il est pratiquement indéréglable. Tous les éléments peuvent être réglés et rendus fixes par collage. L'interféromètre étant très compact et complètement symétrique, il devient possible de loger les deux bras de l'interféromètre dans l'enceinte même du Fabry-Perot; la température et la pression sont alors identiques pour les deux instruments. Ce dernier point est très important pour l'obtention de résultats précis et reproductibles.

2) Souplesse. - Pour que l'étalonnage puisse être aussi serré qu'on le désire, il suffit d'augmenter la longueur L des bras de l'interféromètre; il est également possible d'utiliser du gaz carbonique ou du fréon pour augmenter l'intervalle spectral à explorer. Les franges de Michelson peuvent servir à commander tout système d'asservissement, par exemple un voltmètre digital pour l'enregistrement du spectre point par point, enregistrement qui est alors directement fonction de la différence de marche.

3) L'utilisation de tout autre dispositif interférentiel (Fabry-Perot plan, trou de Young, lames de Jamin, etc.) est moins avantageuse que notre montage, soit au point de vue luminosité, soit au point de vue encombrement. On peut parfaitement concevoir un dispositif d'étalonnage constitué par un laser stabilisé comme source et un Fabry-Perot sphérique de $25 \mathrm{~cm}$. Le dispositif que nous avons adopté est néanmoins beaucoup plus facile à mettre en œuvre et à régler.

Nous sommes très reconnaissants à $\mathrm{P}$. Connes de nous avoir suggéré le montage qui nous a permis de résoudre ce problème d'étalonnage.

Manuscrit reçu le 20 juin 1967.

\section{BIBLIOGRAPHIE}

[1] J ACQUino' (P.) et DUFOUR (Ch.), Journal de Recherches $d u$ C.N.R.S., no 6, 1948.

[2] Chantrel (H.), Colloque International de Spectroscopie, Bellevue, 1957, J. Physique Rad., 1958, 19, 366.

[3] KuHL (J.), Steuder, (A.) et WALTHER (H.), Colloque International "Méthodes nouvelles de spectroscopie instrumentale ", Orsay, 1966, J. Physique, 1967, 28, C2-308.

[4] KUHN (A. G.) et LuCAs-TooTH (H. J.), Colloque International de Spectroscopie, Bellevue, 1957, J. Physique Rad., 1958, 19, 293.

[5] ChaIko (Yu), optics and Spectroscopy, 1966, XX, 5.
[6] BAuche (J.), Diplôme d'Études Supérieures, Paris, 1961.

[7] ROESLER (F. L.) et MACK (J. E.), Phys. Rev., 1964, 135, $1 \mathrm{~A}, 58$.

[8] Connes (P.), Revue d'Optique, 1956, 35, 37-43.

[9] SAUNDERS (J. B.), J. Research N.B.S., 1957, 58, 1.

[10] KoHLER (R. H.), Phys. Rev., 1961, 121, 1104.

[11] Schweitzer (W. G.), J. Opt. Soc. Amer., 1961, 51, 692.

[12] Chabbal, (R.) et Jacquinot (P.), Revue d'Optique, 1961, 40, 15.

[13] Gerstenkorn (S.), Thèse, Paris, Ann. Physique, 1962, 7, 367. 